\title{
Atenção ao idoso frente à pandemia por Covid-19
}

\author{
An alert regarding the elderly who face the \\ Covid-19 pandemic
}

\begin{abstract}
Andreia Clerice da Silva Barbosa ${ }^{1}$, Giseli da Rocha ${ }^{2}$, Maria Goretti David Lopes ${ }^{3}$, Carmen Cristina Moura dos Santos 4

1. ORCID: https://orcid.org/0000-0001-6548-2133. Enfermeira. Universidade Federal do Paraná (UFPR). Secretaria de Estado da Saúde do Paraná (Curitiba, Paraná, Brasil). E-mail: andreia.clerice@sesa.pr.gov.br.

2. ORCID: https://orcid.org/0000-0003-0021-5039. Enfermeira especialista em Gestão do TrabaIho e da Educação no SUS (Escola de Saúde Pública do Paraná). Secretaria de Estado da Saúde do Paraná (Curitiba, Paraná, Brasil). E-mail: giseli.rocha@sesa.pr.gov.br.

3. ORCID: https://orcid.org/0000-0001-7473-6342. Enfermeira. Diretora de Atenção e Vigilância em Saúde da Secretaria de Estado da Saúde do Paraná (Curitiba, Paraná, Brasil).

4. ORCID: https://orcid.org/0000-0003-3162-8346. Enfermeira. Coordenadora da Organização da Rede de Cuidados em Saúde da Secretaria de Estado da Saúde do Paraná (Curitiba, Paraná,
\end{abstract} Brasil).

CONTATO: Flávia Caroline Figel | Endereço: Rua Piquiri, 170 - Rebouças - Curitiba-PR | Telefone: (41) 3330-4526 | E-mail: saudemental@sesa.pr.gov.br

RESUMO A doença Covid-19 atinge com gravidade pessoas acima dos 60 anos, em especial aquelas com comorbidades. A rapidez com que o vírus é transmitido exige conduta rápida na prevenção e adequado manejo, levando em consideração as fragilidades dessa população. Este relato de experiência tem por objeto descrever atividades desenvolvidas pela Divisão de Saúde do Idoso da Secretaria de Estado da Saúde do Paraná, de janeiro a julho de 2020, com vistas a contribuir nas tomadas de decisões de gestores e profissionais de saúde, com enfoque na saúde de idosos institucionalizados. São apresentadas duas Normas Orientativas: Atendimento dos Idosos Frente à Pandemia Covid-19 e Medidas de Prevenção, Controle e Testagem para Covid-19 em Instituição de Longa Permanência para Idosos. Descreve-se a organização e execução de videoconferência e do ciclo de web - Idosos e a Covid-19. A pandemia exige ação e inovação com vistas à proteção do idoso neste momento de risco. 
DESCRITORES: Coronavírus. Pandemias. Idosos. ILPI.

\begin{abstract}
The coronavirus hits more acutely the age group of those over 60 years old, especially those with chronic diseases. The speed at which the virus is transmitted demands a quick response in its prevention and adequate management, keeping in mind the frailties of this age group. This report describes from personal experience the development of the actions taken by the Senior Citizen Health Division of the Health Secretary of the Brazilian state of Paraná from March to July 2020. The objective is to contribute to the decisions of health officials and managers, with the focus on institutionalized senior citizens. The following guidelines are presented: Treatment of seniors facing the Coving-19 pandemic and methods of prevention and Instituting long-term control and testing for Covid-19 for the elderly. Described in this report also is the series of videoconferences regarding seniors and Covid-19. This pandemic demands swift action and innovation to protect the elderly in this time of high risk.
\end{abstract}

DESCRIPTORS: Coronavirus. Pandemics. Aged. Home for the Aged.

\title{
INTRODUÇÃO
}

A

pandemia por Covid-19 tem desestruturado sistemas econômicos, políticos, sociais e, principalmente, o da saúde pública em diversos países e demanda ações rápidas e assertivas. A transmissão do vírus SARS-CoV-2 ocorre no contato com gotículas respiratórias provenientes de pessoas contaminadas ${ }^{1}$, e indiretamente por meio das mãos, objetos ou superfícies².

Segundo evidências, cerca de $80 \%$ das pessoas desenvolvem síndrome gripal com quadro leve, porém 5 a 10\% podem ser acometidas de forma mais grave, com potencial desfecho para insuficiência respiratória, com necessidade de hospitalização e uso de ventilação mecânica, decorrente de evolução para Síndrome Respiratória Aguda Grave, com especial suscetibilidade de determinadas populações ${ }^{3-4}$. Entre os grupos de risco estão pessoas com comorbidades, 
obesos, imunodeprimidos e idosos. Especialmente no grupo de pessoas com idade superior a 60 anos, observa-se maior letalidade, principalmente se existirem comorbidades associadas 5 , como diabetes, hipertensão, doenças cardiovasculares ${ }^{6}$ e síndrome da fragilidade, aumentando as chances de óbito nessa população?

No Paraná, com base nas informações do boletim epidemiológico de 27 de junho de 2020, do total de 562 óbitos por Covid-19, cerca de 70,8\% referem-se à população acima de 60 anos e, destes, aproximadamente 34,9\% entre indivíduos com idade superior a 80 anos. As comorbidades com maiores recorrências, associadas aos casos fatais, foram hipertensão arterial $(25,07 \%)$, diabetes mellitus $(17,20 \%)$ e cardiopatia $(9,33 \%)$, demais 14 agravos somados totalizam $48,40 \%$ dos casos $^{8}$.

Frente à vulnerabilidade neste especial grupo da população, e a realidade estadual nesta pandemia, demandou-se a adoção de medidas para promover sua proteção. Nesse contexto, as Instituições de Longa Permanência para Idosos (ILPI) são locais caracterizados pela concentração de idosos residentes, com diferentes graus de dependência e, muitas vezes, com mais de uma comorbidade associada. As condições de restrição ao leito e dependência de cuidados somadas ao compartilhamento de espaços comuns ${ }^{9}$ constituem situação propícia para rápida e extensa disseminação do vírus SARS-CoV-2.

Nesses ambientes os cuidados são realizados por profissionais de enfermagem e cuidadores de idosos os quais, frequentemente, possuem mais de um vínculo empregatício ${ }^{10}$, aumentando o risco de se tornarem potenciais fontes de dispersão e propagação do vírus, fato que demanda a adoção de medidas especiais de prevenção ${ }^{11}$, reorganização e readequação dos cuidados. Considerando-se este cenário, apresentamos e contextualizamos as ações desenvolvidas pela Secretaria Estadual de Saúde do Paraná (SESA-PR) no enfrentamento à pandemia por Covid-19, com enfoque na atenção aos idosos institucionalizados. 


\section{MÉTODO}

Trata-se de relato de experiência, com abordagem descritiva, das atividades realizadas e documentos de domínio público relativos à SESA-PR e correspondentes à saúde do idoso frente à Covid-19, no período de janeiro a julho de 2020. O conteúdo foi contextualizado frente às publicações do Ministério da Saúde (MS) e da Organização Mundial da Saúde (OMS).

Os autores deste relato compõem a equipe técnica da Diretoria de Atenção e Vigilância em Saúde da SESA-PR. Considerando a Resolução do Conselho Nacional de Saúde (CNS) n 510, de 7 de abril de 2016, foi dispensada a apreciação pelo Comitê de Ética em Pesquisa.

\section{RESULTADOS E DISCUSSÃO}

\section{Nota Orientativa Atendimento dos Idosos Frente à Pandemia Covid-19}

No decorrer do primeiro trimestre de 2020, informações relativas à pandemia, principalmente na Ásia e Europa, com relatos e dados sobre contaminação e alta mortalidade de idosos, se apresentaram de forma progressiva e alarmante, demandando a discussão dos riscos prováveis a este público no Paraná, tão logo ocorresse a primeira transmissão local. Evidenciou-se, portanto, a necessidade de alinhar ações de controle, prevenção e manejo direcionadas aos idosos residentes no Estado, culminando na elaboração e publicação, em 20 de março de 2020, da Nota Orientativa no 4, intitulada "Atendimento dos Idosos Frente à Pandemia Covid-19". Esse documento tem como objetivo orientar as equipes das Secretarias Municipais de Saúde, bem como os profissionais envolvidos nos cuidados aos idosos, incluindo as ILPI ${ }^{12}$.

A Nota contextualiza a atuação dos diferentes níveis de atenção à saúde na assistência à população e apresenta medidas para organização nos atendimentos e monitoramento dos grupos de risco pela Atenção Primária à Saúde (APS) e instrui medidas de prevenção ao coronavírus. O documento também orienta 
a suspensão no atendimento eletivo presencial de idosos na Atenção Ambulatorial Especializada (AAE), apresenta meios alternativos para a continuidade do cuidado e suporte às equipes da APS, recomenda aos profissionais de ILPI medidas de prevenção da transmissão do vírus e afastamento em caso de sintomas respiratórios, além da redução de visitas aos residentes ${ }^{12}$.

São abordados, na referida Nota, os cuidados frente à suspeita de infecção, incluindo recomendações no intuito de evitar o encaminhamento do idoso a serviços de urgência, se possível, atentando para sinais de complicações; e apresenta um direcionamento, baseado no posicionamento da Sociedade Brasileira de Geriatria e Gerontologia (SBGG) e da Sociedade Brasileira de Cardiologia (SBC), sobre o uso de medicamentos durante a pandemia ${ }^{12}$.

\section{Videoconferência}

A população idosa institucionalizada representa o maior grupo de risco para morbimortalidade pela Covid-19, onde idosos frágeis residem em espaços comunitários e têm contato com diversos profissionais que circulam por ambientes considerados de risco, como hospitais, ambulatórios, outras moradias para idosos, transporte coletivo, dentre outros ${ }^{13}$. Importante ressaltar que estas instituições são residências, e muitos dos seus trabalhadores não são profissionais da saúde ${ }^{14}$. Nesse sentido, a SESA-PR identificou a necessidade de realizar a capacitação, com foco nesses trabalhadores, enfatizando as medidas de prevenção e controle da Covid-19, destacando a higiene das mãos, isolamento e limpeza/desinfecção de bancada.

Celeridade no fluxo de informações demandou a realização de um encontro virtual entre gestores e profissionais da saúde, além de trabalhadores e administradores das ILPI. Para viabilizar esta reunião foi utilizada a ferramenta tecnológica de web conferência, o que possibilitou a participação de profissionais de diversos municípios, respeitando a necessidade de distanciamento social e evitando deslocamentos, além da possibilidade de gravação do conteúdo para visualização posterior. As ferramentas utilizadas para a realização do evento 
foram YouTube, por meio do endereço disponibilizado no site da SESA-PR, e via Google Meet, esse último proporcionando interação em tempo real dos participantes. A divulgação da reunião foi realizada com envio de convite por e-mail para todas as 22 Regionais de Saúde, equipes de vigilância sanitária e atenção primária à saúde, além do envio do convite para instituições parceiras, como o Conselho Estadual dos Direitos da Pessoa Idosa, Secretaria Estadual de Justiça, Família e Trabalho, Ministério Público do Paraná, ILPI e ampla divulgação por mídia social (WhatsApp).

Para a organização dos temas a serem abordados foram selecionados assuntos relevantes e relativos à saúde do idoso frente à pandemia. Utilizou-se notas orientativas elaboradas pela SESA-PR, Ministério da Saúde, Agência Nacional de Vigilância Sanitária, artigos e documentos divulgados pela comunidade científica. Os temas em destaque foram Atendimento dos idosos frente à pandemia Covid-19; Vulnerabilidade de idosos institucionalizados; Cuidados de higiene; Adequação dos ambientes e rotinas em ILPI; Uso de Equipamentos de Proteção Individual; Restrição de visitantes; Imunização; e cuidados aos idosos com sintomas gripais.

A videoconferência foi realizada na data de 19 de maio de 2020 e contou com 32 participantes pelo Google Meet e cerca de 150 participantes pelo YouTube; teve duração total de 2 horas e 45 minutos. O número de acessos na data de 10 de julho de 2020 foi de 1.782 visualizações.

As dificuldades encontradas foram a falta de conhecimento e habilidade no uso dos recursos da internet, e indisponibilidade de câmera e microfone em muitos dos computadores da SESA-PR. Foi possível a troca de experiências, esclarecimento de dúvidas e compartilhamento de expectativas e práticas, bem como viabilizou-se o repasse de informações técnicas. Como aprendizado, a experiência oportunizou uma nova alternativa para a realização de eventos, com possibilidades de alcançar diversos profissionais em diferentes localidades. Constatou-se a viabilidade de participação de indivíduos e equipes que, devido às características do trabalho assistencial contínuo, não conseguiriam 
se deslocar para uma reunião presencial. Foi evidenciada a integração intra e interinstitucional em prol da população idosa, não obstante, o uso desta ferramenta permite rever o evento no YouTube através do canal da SESA-PR, acessando o título "Medidas de Prevenção e Controle para a COVID-ILPI", ou através do link: https://www.youtube.com/watch?v=dTswQ0bBVAQ.

\section{Ciclo de Web - Idosos e a Covid-19}

A pandemia evidenciou as fragilidades na saúde e nos cuidados aos idosos, principalmente nos institucionalizados. Ficou evidente que a capacitação de profissionais engajados na assistência a essa população é imprescindível para um atendimento eficaz e seguro, não só durante a pandemia, mas sendo estendido para momentos posteriores ${ }^{15}$. Sendo assim foi elaborado um conjunto de web videoconferências, intituladas "O Idoso e a Covid-19".

O ciclo de web conferências foi realizado pela SESA-PR em cooperação do Conselho Nacional dos Secretários de Saúde. A iniciativa teve como objetivo ampla discussão de assuntos pré-selecionados, relevantes no contexto assistencial do idoso, com informações atualizadas e evidências científicas no que concerne ao tema, com intuito de capacitação de gestores e profissionais da saúde, todos os níveis de atenção, administradores e trabalhadores de ILPI sobre os cuidados ao idoso frente à pandemia.

A data de início das videoconferências foi 9 de junho de 2020 com previsão de término em 28 de julho de 2020. Os assuntos expostos contemplam o cuidado ao idoso na pandemia, cuidado ao idoso institucionalizado, a estratificação de risco e a proporcionalidade terapêutica, e o idoso na Rede de Atenção à Saúde. Os encontros virtuais são realizados semanalmente, às terças-feiras, entre 15 e 17 horas, com temas apresentados por conferencistas especialistas e convidados da Rede de Atenção à Saúde para discussão de casos clínicos. Os canais de comunicação utilizados são o YouTube e o Google e a divulgação é feita pela página eletrônica da SESA-PR e apoio das Regionais de Saúde.

\section{Nota Orientativa sobre Medidas de Prevenção, Controle e Testagem}




\section{para Covid-19 nas ILPI}

As ILPIs requerem estratégias que culminem tanto na prevenção e proteção dos idosos residentes como a dos trabalhadores dessas instituições. Nesse sentido, um trabalho integrado da Divisão de Vigilância Sanitária de Serviços e da Divisão de Atenção ao Idoso culminou na elaboração da Nota Orientativa $n^{\circ}$ 41/2020, intitulada "Medidas de Prevenção, Controle e Testagem para Covid-19 nas Instituições de Longa Permanência para Idosos (ILPI)", de 03 de julho de 2020. Nela são relacionadas diversas orientações que abrangem os gestores municipais, as unidades básicas de saúde, dirigentes e profissionais das ILPI. Descreve-se os sinais e sintomas característicos da Covid-19, destacando a importância de conhecer as especificidades na população idosa, bem como a necessidade de indicação do ponto de Rede de Atenção à Saúde que será de referência para a ILPI ${ }^{16}$.

Outra estratégia proposta na referida Nota é a elaboração do Plano de Contingência Institucional ( $\mathrm{PCI}$ ) pelas ILPI, o qual contribui para que medidas adotadas para o enfrentamento ao coronavírus sejam padronizadas, estruturadas e agilizadas, objetivando a prevenção e redução da propagação do vírus, por meio da definição de diretrizes, normas e orientações ${ }^{17}$. Essa servirá também como diretriz nas tomadas de decisões frente às necessidades decorrentes de casos da doença dentro da ILPI. Para tanto, deverá contemplar fluxos, protocolos, instruções, indicações de encaminhamentos em casos de agravamento, provisão de equipamentos de proteção individual, bem como, permite prever insumos necessários para a execução das atividades e definição de profissional responsável pela implementação das ações de prevenção e controle da doença na instituição. As atividades designadas a este profissional estão descritas na Nota.

Outra estratégia contemplada no documento se refere ao rastreamento de casos de Covid-19 nas ILPI. Segundo evidências, pessoas com quadro leve da doença ou mesmo assintomáticas são transmissoras do vírus, tornando-se um grande desafio na detecção e interrupção da propagação da doença, principal- 
mente se ocorrer dentro das ILPI, locais com grande fluxo de idosos e profissionais. Sendo assim, para mitigação das complicações de disseminação do vírus dentro dessas instituições, uma das estratégias é o rastreamento laboratorial de idosos e trabalhadores, ao longo da pandemia ${ }^{13}$.

Na tentativa de implementar ações que reduzam os agravos nessa população, foi incluído na Nota Orientativa a estratégia de rastreamento laboratorial de idosos e colaboradores. Para tal, deverá ser realizado, inicialmente, o teste por RT-PCR (Reação Transcriptase Reversa em tempo real), o qual confirma a existência da doença através da presença de material genético do vírus ${ }^{18}$, auxiliando no levantamento de casos, mesmo que assintomáticos. Após essa coleta, a Nota recomenda o monitoramento por teste rápido e, ocorrendo casos positivos, é necessário realizar novamente RT-PCR ${ }^{16}$.

São recomendadas medidas de isolamento do idoso e afastamento do trabalhador, quando resultado positivo, suspendendo a transmissão. Destaca-se a relevância da articulação entre gestores municipais, ILPI, Regionais de Saúde e Assistência Social, para realização da testagem. Foram contempladas, ainda, ações necessárias para a efetivação do isolamento, seja por diagnóstico de Covid-19 ou por admissão de novo residente na instituição; condução correta em caso de agravamento do quadro clínico; organização e adequação do ambiente; restrição e estabelecimento de regras para as visitas em casos de extrema necessidade ${ }^{16}$.

\section{CONSIDERAÇÕES FINAIS}

A pandemia expôs a fragilidade e particularidades de populações especialmente vulneráveis, como a dos idosos, e a construção das estratégias desenvolvidas foi embasada em abordagem ampla e resoluta. Concomitantemente, possíveis obstáculos, como o distanciamento geográfico, restrição de tempo 
e despesas com deslocamentos, puderam ser superados por meio do uso de ferramentas tecnológicas, as quais também contribuíram para a troca de experiências e construção do cuidado seguro e eficaz.

Cientes de que as diretrizes elaboradas necessitam ser amplamente compartilhadas, a capacitação é reconhecidamente essencial para a efetivação das ações, e determinante também na consolidação de vínculos entre os diversos profissionais.

A dinâmica da pandemia e de resultados de estudos demandam à área técnica de Atenção ao Idoso contínua ação e inovação, com base nas evidências científicas e na epidemiologia da doença, reformulação das recomendações e elaboração de novas diretrizes com vistas à segurança e proteção ao idoso.

\section{AGRADECIMENTOS}

Agradecemos à Elaine Drehmer de Almeida Cruz, apoiadora pela OPAS junto à SESA-PR, pelas contribuições ao texto.

\section{REFERÊNCIAS}

1. Organização Pan-Americana da Saúde (OPAS). Folha informativa - COVID-19 (doença causada pelo novo coronavírus) [Internet]. Brasília: 2020. [citado em 2020 Mai 27]. Disponível em: https://www.paho.org/bra/index.php?option=com content\&view=article\&id=6101:covid19\&ltemid=875.

2. Agência Nacional de Vigilância Sanitária (BR). Nota Técnica n 4/2020-GVIMS/GGTES/ANVISA - Orientações para Serviços de Saúde: Medidas de Prevenção e Controle que devem ser adotadas durante a Assistência aos casos Suspeitos ou Confirmados de Infecção pelo Novo Coronavírus (SARS-COv-2). Brasília: Anvisa, 2020.

3. Ministério da Saúde (BR). Protocolo de Manejo Clínico da COVID-19 na Atenção Especializada. 1. ed. revisada. Brasília: Ministério da Saúde, 2020.

4. Ministério da Saúde (BR). Protocolo de Manejo Clínico do Coronavírus (COVID-19) na Atenção Primária à Saúde - versão 9. Brasília: Ministério da Saúde, 2020.

5. Agência Nacional de Vigilância Sanitária (BR). Nota Técnica n05/2020 - GVIMS/GGTES/ANVISA - Orientações para a Prevenção e o Controle de Infecções pelo Novo Coronavírus (SARS-CoV-2) em Instituição de Longa Permanência para Idosos (ILPI). Brasília: Anvisa, 2020

6. Zhou F, Yu T, Du R, Fan G, Liu Y, Liu Z et al. Clinical course and risk factors for mortality of adult inpatients with COVID-19 in Wuhan, China: a retrospective cohort study [Internet]. Lancet; 2020. 395(10229):1054- 62. [citado em 2020 Jun 27 ]. Disponível em: https://www.thelancet.com/pdfs/journals/lancet/PIIS0140-6736(20)30566-3.pdf.

7. Boas PJFV, Uehara CA, Bremenkamp MG, Roriz JS Filho, Kairalla MC, Gomes DCA et al. Recomendações para prevenção e 
controle de infecções por coronavírus (SARS-CoV-2) em instituições de longa permanência para idosos (ILPI). Seção especial - Posicionamentos da SBGG diante da pandemia COVID-19 [Internet]. Sociedade Brasileira de Geriatria e Gerontologia (SBGG); 2020. [citado em 2020 Jun 27]. Disponível em: https://cdn.publisher.gn1.link/ggaging.com/pdf/GGA_142ESP3_PT_ AOP.pdf.

8. Secretaria da Saúde do Estado do Paraná (SESA/PR) - Informe Epidemiológico - Informe Epidemiológico [Internet]. 2020. [citado em 2020 Jun 27]. Disponível em: http://www.saude.pr.gov.br/sites/default/arquivos_restritos/files/ documento/2020-06/informe_epidemiologico_27_06_2020.pdf.

9. Nunes VMA, Machado FCA, Morais MM, Costa LA, Nascimento ICS, Nobre TTX et al. Covid-19 e o cuidados de idosos Recomendações para Instituições de Longa Permanência [Internet]. Natal: EDUFRN; 2020. [citado em 2020 Jul 5]. Disponível em: https://repositorio.ufrn.br/jspui/handle/123456789/28754.

10. Silva BM, Lima FRF, Farias FSAB, Campos ACS. Jornada de trabalho: Fator que interfere na qualidade da assistência de enfermagem. Texto e contexto Enfermagem [revista em internet]. Fortaleza: 2006. [citado em 2020 Jun 12]; vol.15, n.3, pp.442-448. Fortaleza, CE. Disponível em: https://www.scielo.br/pdf/tce/v15n3/v15n3a08.pdf.

11. Agência Nacional de Vigilância Sanitária (BR). Nota Técnica n07/2020-GVIMS/GGTES/ANVISA - Orientações para a prevenção da Transmissão de COVID-19 dentro dos serviços de saúde [Internet]. Brasília, 2020. [citado em 2020 Mai 27]. Disponível em: http://portal.anvisa.gov.br/documents/33852/271858/NOTA+T\%C3\%89CNICA+-GIMS-GGTESANVISA+N\%C2\%BA+07-2020/f487f506-1eba-451f-bccd-06b8f1 b0fed6.

12. Secretaria da Saúde do Estado do Paraná (SESA PR). Nota Orientativa $n^{\circ} 4$ - Atendimento dos Idosos frente à Pandemia COVID-19. Edição 20 mar 2020. Curitiba: SESA; 2020. Disponível em: http://www.saude.pr.gov.br/sites/default/arquivos_ restritos/files/documento/2020-06/no_04_atendimento_dos_idosos_frente_a_pandemia_covid_19.pdf.

13. Moraes EN, Viana LG, Resende LMH, Vasconcellos LS, Moura AS, Menezes A et al. COVID-19 nas Instituições de Longa Permanência para Idosos: Estratégias de rastreamento laboratorial e prevenção da propagação da doença. Revista da Associação de Saúde Coletiva. [Internet]. Rio de Janeiro: Ciênc. saúde coletiva; 2020. [citado em 2020 Jun 15]. Disponível em: http://www.cienciaesaudecoletiva.com.br/artigos/covid19-nas-instituicoes-de-longa-permanencia-para-idosos-estrategiasde-rastreamento-laboratorial-e-prevencao-da-propagacao-da-doenca/17631 ?id=17631\&id=17631\&id=17631.

14. Camarano AA, Mello JL, Pasinato MTM, Kornis GEM, Kanso S, Duarte YAO et al. Cuidados de longa duração para a população idosa: um novo risco social a ser assumido? [Internet]. Rio de Janeiro: Ipea, 2010. 350 p. [citado em 2020 Jun 29]. Disponível em: https://www.ipea.gov.br/portal/images/stories/PDFs/livros/livro_cuidados.pdf.

15. Hammerschmidt KSA, Santana RF. Saúde do idoso em tempos de pandemia Covid-19 [Internet]. Cogitare enferm.; 2020. [citado em 10 Jul 2020]; 25. Disponível em: https://revistas.ufpr.br/cogitare/article/view/72849.

16. Secretaria da Saúde do Estado do Paraná. Nota Orientativa n 41 - Medidas de prevenção, controle e testagem para COVID-19 nas Instituições de Longa Permanência para Idosos (ILPI) [Internet]. Paraná: SESA; 2020. Disponível em: http:// www.saude.pr.gov.br/sites/default/arquivos_restritos/files/documento/2020-07/NO_41_MEDIDAS_DE_PREVENCAO_ CONTROLE_E_TESTAGEM_PARA_COVID_19_NAS_\%2OINSTITUICOES_DE_LONGA_PERMANENCIA_PARA_IDOSOS_V1.pdf.

17. Conselho Regional de Enfermagem da Bahia (COREN BA). Roteiro para Elaboração do Plano de Contingência dos serviços de saúde para enfrentamento da COVID-19 [Internet]. Bahia: COREN-BA; 2020. [citado em 2020 Jun 16]. Disponível em: http://ba.corens.portalcofen.gov.br/roteiro-para-elaboracao-do-plano-de-contingencia-dos-servicos-de-saude-paraenfrentamento-do-covid-19_55228.html/print/.

18. Sociedade Brasileira de Patologia Clínica. Métodos Laboratoriais para Diagnóstico da Infecção pelo SARS-CoV-2. Recomendações SBPC ML. Rio de Janeiro; 2020. [citado em 2020 Jul 7]. Disponível em: http://www.sbpc.org.br/wp-content/ uploads/2020/04/MetodosLaboratoriaisDiagnosticoSARS-CoV-2.pdf.

\section{RECEBIDO:31/07/2020 \\ ACEITO: 13/10/2020}

